\title{
THE CONVERSION OF SURF II TO SURF III
}

\author{
M.L. Furst", R.M. Graves, A. Hamilton, L.R. Hughey, R.P. Madden ${ }^{+}$, R.E. Vest, NIST, \\ Gaithersburg, MD, W.S. Trzeciak, R.A. Bosch, Synchrotron Radiation Center, Stoughton, WI, \\ L. Greenler, P. Robl, D. Wahl, Physical Sciences Laboratory, Stoughton, WI
}

\section{Abstract}

The Electron and Optical Physics Division of the Physics Laboratory has operated the Synchrotron Ultraviolet Radiation Facility (SURF) at the National Institute of Standards and Technology (NIST) for over 30 years. Initially operated in a parasitic mode at the NIST electron synchrotron, the facility was converted into the SURF II electron storage ring in 1974. Since then, SURF II has been the United States national standard of irradiance in the vacuum ultraviolet spectral region, but the radiometric accuracy of SURF II was limited by the uniformity of the magnetic field encountered by electrons as they traversed their orbit. Variations of up to $0.5 \%$ limited the knowledge of the local bending radius at any tangent point and restricted the accuracy of the irradiance calculations. To improve the radiometric accuracy of SURF, an entirely new magnet structure was installed in the summer of 1998. The azimuthal uniformity of the SURF III magnetic field has been improved by a factor of 50 at $388 \mathrm{MeV}$ and a factor of 25 at $260 \mathrm{MeV}$, allowing irradiance calculations to be made with much higher accuracy than SURF II could achieve. As an additional benefit, the use of improved magnet material, a smaller air gap between the poles, and new magnet windings has increased the magnetic field strength at the electron orbit, allowing SURF III to store electrons at energies as high as $400 \mathrm{MeV}$, compared to $300 \mathrm{MeV}$ for SURF II. The higher electron energy will extend the usable range of radiation from SURF to shorter wavelengths, enabling experiments in the "water window" from $2.3 \mathrm{~nm}$ to $4.4 \mathrm{~nm}$. We present here the major design goals and features of the SURF III magnet, the results of magnet field mapping and other measurements, and the facility performance to date.

\section{BACKGROUND}

The NIST Synchrotron Ultraviolet Radiation Facility (SURF) began as an electron synchrotron operating at $180 \mathrm{MeV}$ in 1961. In 1962 the synchrotron was modified to study properties of the synchrotron radiation continuum and construction was begun on instrumentation for optical experiments using this radiation. The advantages of an electron storage ring design for spectroscopy and radiometry led to the conversion to the SURF II electron

\footnotetext{
${ }^{\#}$ Email: furst@nist.gov

${ }^{+}$R.P. Madden is retired from NIST
}

storage ring in 1974 [1]. SURF II operated from 1974 to 1997 over an energy range from $140 \mathrm{MeV}$ to $300 \mathrm{MeV}$ with stored beam currents as high as $390 \mathrm{~mA}$. Most of the magnet structure was not modified for the conversion to a storage ring. The upper and lower yokes, backlegs, and upper and lower poles remained the original laminated steel used for the synchrotron. A laminated design limited eddy current effects in the synchrotron electromagnet operating at $60 \mathrm{~Hz}$. In order to achieve suitable operating characteristics of the magnetic field for a storage ring, five correction coils and new main coils were designed and manufactured by the University of Wisconsin for the SURF II configuration. Despite limitations in the laminar design, careful assembly of the electromagnet, alignment of the poles, and accurate measurement of the appropriate parameters resulted in SURF II becoming a primary standard source of irradiance in the UV and VUV. Ultimately the relative uncertainties in irradiance at $284 \mathrm{MeV}$ (combined standard uncertainties with a coverage factor of two) ranged from $\pm 9.0 \%$ at $4 \mathrm{~nm}$ to $\pm 0.8 \%$ at $400 \mathrm{~nm}$.

\section{A NEW DESIGN}

With an eye towards expanding the role of SURF in national radiometric standards, personnel at the University of Wisconsin-Madison (UW-Madison) Physical Sciences Laboratory (PSL) and Synchrotron Radiation Center (SRC) were approached to design a new electromagnet. Using POISSON and ANSYS programming codes, the UW-Madison designers, based on information gathered from modeling the old electromagnet, developed a new design using modern solid 1008 magnet steel. It was decided to use solid 1008 steel for the entire magnet structure to take advantage of its higher saturation level compared to the old steel and to increase the density of steel by filling in the gaps of the laminated structure. With these changes and a smaller pole gap, operation at an energy near $400 \mathrm{MeV}$ would become a possibility. Because of concerns about eddy current creation in the poles during rapid ramping of the magnetic field from injection field to operating field, it was decided to make each pole in two $\mathrm{C}$-shaped segments. The poles were then assembled onto a backing plate with a $76 \mu \mathrm{m}$ thick mylar shim between the two segments. The general layout of the magnet steel is shown in Figure 1 which shows a N-S cross section through the magnet.

There are five blocks comprising each of the upper and lower yokes that are held together with four horizontal tie 
rods. These blocks, each weighing about $134 \mathrm{kN}$ (15 ton), were aligned using leveling jacks to within $25 \mu \mathrm{m}$ of each other.

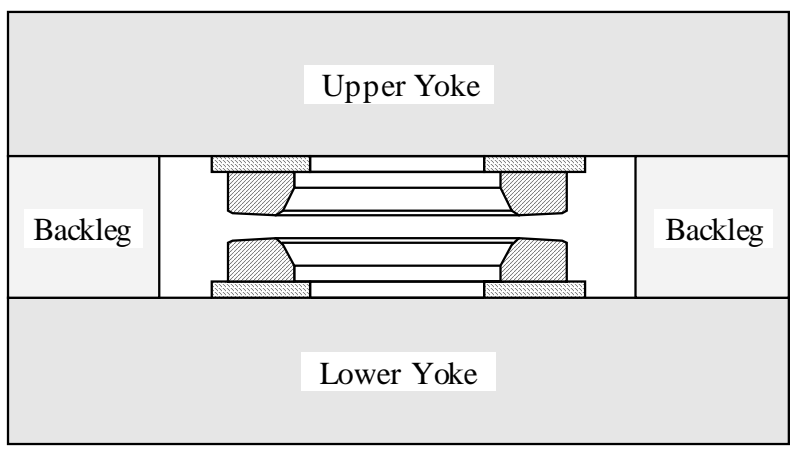

Figure 1: Cross-sectional view of SURF III magnet steel including upper and lower poles.

At the factory, the entire steel structure was assembled, precision holes were drilled between blocks, and then locating pins were inserted. The structure was then disassembled for shipping. The cylindrical hardened steel locating pins were manufactured to a tolerance of $2.5 \mu \mathrm{m}$ and used to reassemble the structure with the proper alignment. Once the lower yoke was assembled on the leveling jacks, the assembly was lifted into place on a support structure so that its geometric center was the same as that of the lower magnetic pole of SURF II to within $0.25 \mathrm{~mm}$. At the factory two blocks were welded together to form a backleg weighing about $128 \mathrm{kN}$ (14.4 ton). The two backlegs were placed on top of the lower yoke again with the aid of locating pins. The lower pole was then placed on the lower yoke and centered. A dial indicator was attached to an arm bolted to an air bearing rotary table to measure the alignment of the poles. Each pole has precision horizontal and vertical fiducial surfaces machined near its perimeter. By mapping the dial indicator readings of these surfaces, the level and centering of the poles could be determined. Sample centering and level maps for the lower pole are shown in Figure 2.

A set of 10 pie-shaped azimuthally equidistant spacers were attached to each pole backing plate to provide about $7.4 \mathrm{~mm}$ of gap between each backing plate and its yoke. In between each pair of spacers a pie-shaped shim carrier was inserted capable of carrying shims up to $0.5 \mathrm{~mm}$ thick. These shims were used to make small adjustments to the azimuthal uniformity of the magnetic field. Once the upper pole was mounted to the upper yoke we were able to measure the gap between the poles directly with an inside micrometer. After initial measurements revealed the poles were slightly nonparallel, the pole gap was adjusted by adding shims between the tops of the backlegs and the upper yoke. The resulting map of the pole gap measured between upper and lower horizontal fiducial surfaces is shown in Figure 3. After the poles were aligned, new main coils were mounted and centered on the poles. A pair of upper and lower trim coils were included in the design to allow for adjustment of the radial field index, defined as:

$$
n \equiv-\frac{r}{B} \frac{\partial B}{\partial r}
$$

Where $r$ is the radius and $B$ is the magnetic flux density.

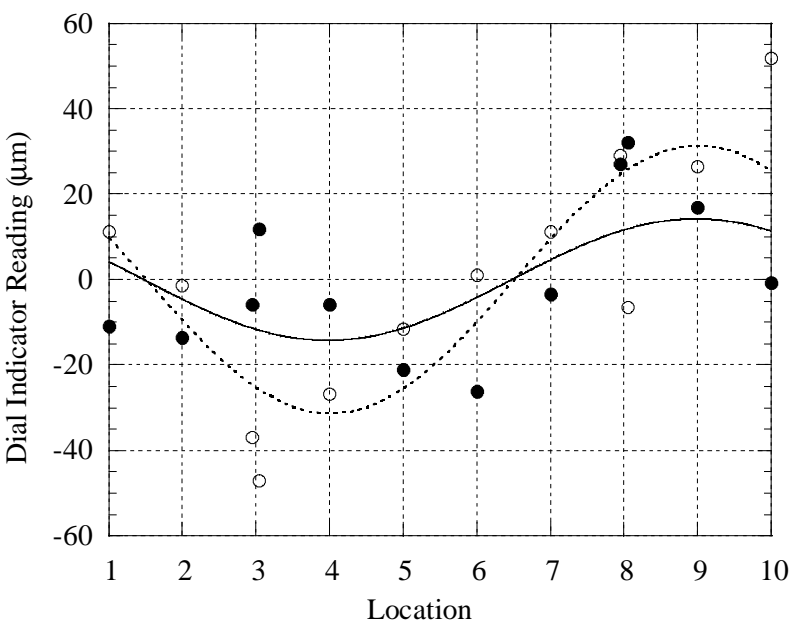

Figure 2: Centering (solid line with solid circles) and level (dashed line with open circles) of the lower pole. The points are deviations from the average of 10 measurements of the vertical (for centering) and horizontal (for level) fiducial surfaces at approximately $36^{\circ}$ intervals, location 3 being due south. The lines show fits to the equation:

$$
y=A \cos [36(x-\theta)]
$$

Where $\mathrm{A}$ is the amplitude of the cosine wave and $\theta$ is the phase, their respective values being $14.2 \mu \mathrm{m}$ and 9.0 for centering and $31.4 \mu \mathrm{m}$ and 9.0 for level.

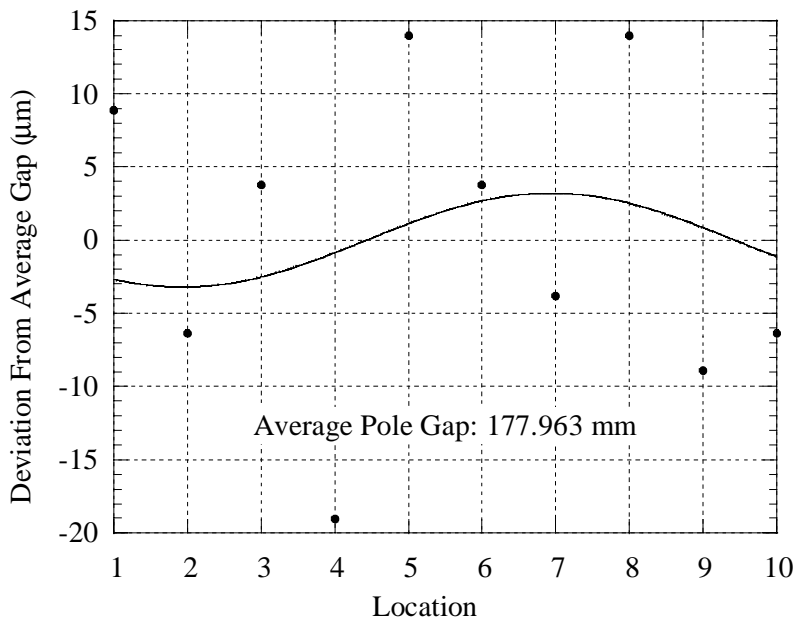

Figure 3: Parallelism of the upper and lower poles as measured by the distance between upper and lower horizontal fiducial surfaces. The coefficients of the cosine curve fit are $3.2 \mu \mathrm{m}$ and 6.9. (See Fig. 2) 
To operate in the storage ring mode, the index is required to be less than 0.75 . The new magnet was designed to have an index of 0.6 to avoid resonant coupling of the horizontal and vertical betatron tunes at values of 0.56 and 0.64. Also an index near 0.6 provides a reasonable balance between radial and longitudinal damping of the electron beam. The trim coils were included to provide for small adjustments of the index during ramping to final energy and possibly at the highest energies where the index was expected to have a significant radial gradient. During operation of the storage ring for radiometric experiments the trim coils would be disconnected.

The new design also included some modifications to the storage ring vacuum chamber. Two new ports were added to accommodate new beamlines that pass through holes cut through the backleg steel. One magnetic field probe port was added, and two older ports were modified to accommodate magnetic field probes at approximately $120^{\circ}$ intervals around the orbit.

\section{MAGNETIC FIELD MEASUREMENTS}

In order to measure the ability of the new electromagnet to provide a perfect circular orbit and the appropriate index profile, a large number of magnetic field maps were obtained. UW-Madison provided us with a computerdriven precision field mapping system capable of measuring field variations of about $0.003 \%$. Magnetic field probes were constrained to move in measurable independent azimuthal and radial increments relative to the fiducial surfaces. Also, the probes were set to move in the midplane between the poles. The absolute accuracy of the knowledge of probe positions was approximately $0.2 \mathrm{~mm}$.

Azimuthal field maps were obtained at the orbital radius over a range of main coil currents from 18.5 A to $900 \mathrm{~A}$, corresponding to magnetic flux densities of $0.04 \mathrm{~T}$ to $1.66 \mathrm{~T}$ and equivalent to electron energies from $10 \mathrm{MeV}$ to $417 \mathrm{MeV}$. After optimizing the configuration of the shims in the shim carriers and adding poleface shims at the joints between the pole segments, the azimuthal field maps showed variations no greater than $\pm 0.03 \%$ from equivalent energies of $52 \mathrm{MeV}$ to $417 \mathrm{MeV}$. Figure 4 shows the results of shim optimization with $700 \mathrm{~A}$ in the main coils. Figure 5 shows a comparison of the azimuthal field uniformity for SURF II at its nominal operating energy of $284 \mathrm{MeV}$ with SURF III at its initial expected operating energy of $331 \mathrm{MeV}$. The average maximum deviation for SURF II was $\pm 0.686 \%$ whereas for SURF III it is $\pm 0.014 \%$. We expect to realize a magnetic field uncertainty of $\pm 0.05 \%$ at the $331 \mathrm{MeV}$ initial operating energy. The contribution of this component to the total uncertainty in irradiance at a wavelength of $4 \mathrm{~nm}$ would be $\pm 0.4 \%$ as compared to $\pm 9.0 \%$ for the contribution from the $\pm 0.6 \%$ uncertainty of SURF II.

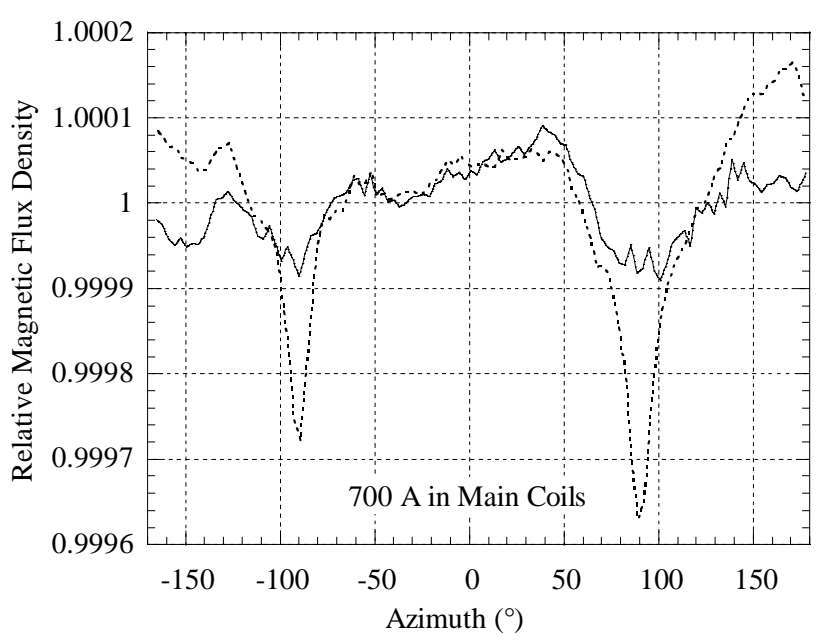

Figure 4: Results of shim optimization on magnetic field azimuthal uniformity at $700 \mathrm{~A}$ (equivalent to $352 \mathrm{MeV}$ ). The dotted line shows normalized field measurements with identical shims in the twenty shim carriers and no poleface shims. The solid line displays measurements after adjusting the shim configuration and installing poleface shims.

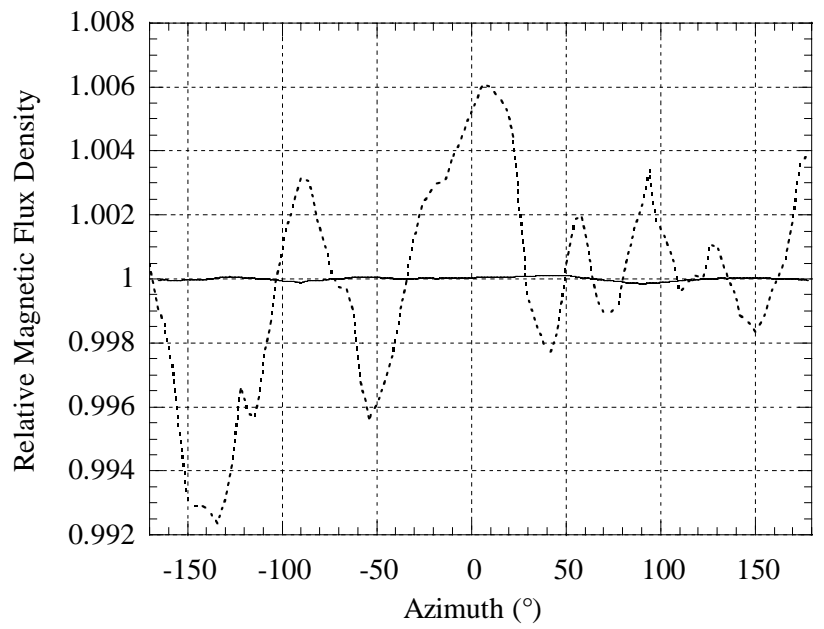

Figure 5: Comparison of azimuthal field maps of SURF II at $284 \mathrm{MeV}$ operating energy (dotted line) and SURF III at initial $331 \mathrm{MeV}$ operating energy (solid line).

We gratefully acknowledge the help of Dave Eisert and Don Holly from UW-Madison, Ping-Shine Shaw from NIST, and Rebecca Friedman from Union College.

\section{REFERENCES}

[1] E.M. Rowe, et al, "The Conversion of the NBS $180 \mathrm{MeV}$ Synchrotron to a $240 \mathrm{MeV}$ Electron Storage Ring for Synchrotron Radiation Research," Proceedings of the $9^{\text {th }}$ International Conference on High Energy Accelerators, SLAC, Stanford, CA, May 1974. 